benefit great enough to outweigh the possible risk of sensitising the patient to the antibiotic or of adding new recruits to the army of antibiotic-resistant strains of bacteria ? It has proved surprisingly difficult to answer these questions acccurately.

The lesions of eczema, particularly in atopic subjects, are very readily colonised by staphylococci, and even the normal skin of patients with eczema carries a larger bacterial population than that of controls. It has been assumed that the moist conditions in the lesions favour bacterial multiplication and that treatment should be "directed at the skin lesions rather than at eradication of microorganisms." Eczema does indeed favour colonisation and multiplication, but the critical question is whether such colonisation increases the severity or duration of the lesions so that they can logically be regarded as "infected." Kligman and his colleagues in Philadelphia ${ }^{2}$ have proposed that "a lesion can be said to be infected when the burden of Staphylococcus aureus is great enough to add to morbidity in some demonstrable way." In a series of quantitative investigations ${ }^{3}$ they found that the lesions in children with atopic dermatitis improved appreciably after the application of neomycin $1 \%$ only when the pretreatment burden was over $10^{6}$ Staph aureus $/ \mathrm{cm}^{2}$. They concluded that Staph aureus is likely to be aggravating the primary disease when the count exceeds this figure. In further studies, in which the bacterial population of lesions was increased by occluding the lesions with polyethylene, they obtained additional support for this conclusion. The authors emphasise the important practical observation that with a staphylococcal load of $10^{6} / \mathrm{cm}^{2}$ there is no clinical evidence of infection: crusting and pus develop only with very much higher counts. The results of symmetrical paired comparison trials that failed to show any significant benefit from the addition of neomycin to a corticosteroid ${ }^{4}$ are not valid because effective amounts of antibiotic can be transferred on the fingers from the treated to the untreated side. ${ }^{5}$

Neomycin is the antibiotic most often used in proprietary combinations with a corticosteroid, but it has a bad reputation among dermatologists as a contact sensitiser. A critical review of published reports ${ }^{2}$ suggests that the risk of contact dermatitis is important, especially in eczema of the lower leg and when application is long continued. The short-term application of neomycin, particularly in children, seems, however, to carry little risk of sensitisation.

The risk of inducing neomycin-resistant strains of Staphylococcus is certainly a consideration in closed communities, but the evidence leads Leyden and Kligman ${ }^{2}$ to the conclusion that in outpatient practice (and in general practice) the risk is very small. This recent paper by Leyden and Kligman, ${ }^{2}$ the latest report on an important and continuing research project, provides invaluable guidance for the practitioner. Moreover, the findings have been fully confirmed by other workers in a multicentre clinical trial. ${ }^{6}$ Recommendations for the scientific management of secondarily infected dermatoses may be summarised as follows:

Firstly, the secondary infection of eczema may be clinically evident as impetiginous crusting or pustulation, but substantial infection may none the less be present in the absence of such changes. It is seldom possible to obtain quantitative bacteriological reports as a routine procedure, but semi-quantitative reports are often provided. Clinical evidence of infection or a report that Staph aureus is present in abundance will justify prescribing a corticosteroid-antibiotic combination.

Secondly, for dermatoses infected with Staph aureus neomycin will usually be the antibiotic of choice. The corticosteroid-antibiotic combination should be applied for no longer than seven to 10 days, after which it may, if necessary, be replaced by a corticosteroid alone. Intermittent resumption of the combination may be desirable, particularly in atopic children. But prolonged continuous application should be avoided, as should any application of neomycin to eczema or ulcers of the lower leg.

Lastly, combinations of corticosteroids with other antibiotics or antimicrobial agents may be indicated if Gramnegative rods or candida are present. The same general principles hold good: the combination should be applied for only a short period, after which it may be replaced, if necessary, by a steroid alone.

1 Noble, W C, and Somerville, D A, Microbiology of Human Skin, p 256. London, Saunders, 1974.

2 Leyden, J J, and Kligman, A M, British fournal of Dermatology, 1977, 96, 179.

${ }^{3}$ Leyden, J J, Marples, R, and Kligman, A M, British fournal of Dermatology, $1974,90,525$.

${ }^{4}$ Davis, C M, Fulghum, D D, and Taplin, D, Fournal of the American Medical Association, 1968, 203, 298.

5 Marples, R R, and Kligman, A M, British fournal of Dermatology, 1973, 88, 61 .

${ }^{6}$ Wachs, G N, and Maibach, H, British fournal of Dermatology, 1976, 95, 323.

\section{Food and the handling of drugs}

Food and nutritional state have recently been recognised as important factors in both the absorption and metabolism of drugs. Drugs given by mouth are absorbed mainly from the small intestine, where a large surface area facilitates passive diffusion, and the rate of drug absorption depends mainly on the rate of gastric emptying. ${ }^{2}$ The presence of food in the stomach delays the speed with which drugs reach the general circulation and postpones the effects of those (such as analgesics and hypnotics) that are meant to have immediate actions. This effect does not matter when drugs are given repetitively, because the rate (as opposed to the extent) of absorption has no influence on "steady-state" concentrations during long-term treatment. ${ }^{3}$ Indeed, administration with food may be helpful for patients taking drugs that cause local irritation of the gastric mucosa. Despite this generalisation, a few drugs (such as acid-labile penicillins) are destroyed in the acid environment of the stomach or are metabolised by the gastric mucosa ${ }^{4}$ (levodopa): in these instances food may reduce both the rate and the extent of absorption. ${ }^{2}$

A few drugs which resemble certain dietary constituents undergo active transport across the small intestinal mucosa. Of those, $\alpha$-methyldopa and levodop $\mathrm{a}^{5}$ are the most important, and though high protein diets might be expected to reduce their effectiveness (by competition for active transport with other amino-acids) no systematic studies appear to have been undertaken.

Many drugs are completely absorbed from the gastrointestinal tract but are then extensively metabolised in the small intestinal wall or the liver before reaching the general circulation. This process ${ }^{6}$ of "first-pass elimination" is known to reduce the systemic availability of propranolol, oxprenolol, isoprenaline, lignocaine, aspirin, and paracetamol. In rats the activities of drug oxidising enzymes in the intestinal mucosa have been shown ${ }^{78}$ to be increased by feeding the animals with sprouts, cauliflower, and other vegetables as well as by benzo(a)pyrine-a constituent of both cigarette smoke and charcoal-grilled meat. Recently, Conney and 
his colleagues ${ }^{9}$ found that a hospital diet supplemented with charcoal-grilled beef lowered plasma levels of phenacetin in man, probably by increasing first-pass elimination in the intestinal mucosa or the liver.

Once a drug has reached the general circulation a major determinant of the intensity and duration of its effect is its rate of elimination. ${ }^{10}$ Many drugs are oxidised in the endoplasmic reticulum of the liver cell, and normal individuals vary widely in the rates at which this occurs. ${ }^{10}$ The pioneering twin studies of Vesell and Page ${ }^{11}$ in the United States and the late Balzar Alexanderson and his colleagues ${ }^{12}$ in Sweden showed that polygenic factors influenced an individual's capacity to oxidise drugs. A family study in Britain by Whittaker and Price Evans ${ }^{13}$ suggested that the heritability of phenylbutazone oxidation was 0.65-but also reported a significant correlation of phenylbutazone half lives between unrelated spouses, confirming the importance of environmental factors.

The nature of some of these environmental influences are becoming clearer: apart from the known "inducing" effects of certain drugs, insecticides, and cigarette smoke, diet is also relevant. The half life of antipyrine (a widely used model substrate for drug oxidation) is slightly shorter in people who drink a lot of coffee. ${ }^{14}$ Of greater practical importance are the findings of Kappas and his colleagues, ${ }^{15}$ who found that changing normal volunteers' diets to a low carbohydrate-high protein mix caused substantial reductions in the half lives of antipyrine and tolbutamide (another oxidised drug); and that the half lives of these drugs could be restored by feeding a high carbohydrate-low protein diet. Fraser and his colleagues ${ }^{16}$ have shown that Asjan vegetarians living in London have significantly longer antipyrine half lives than do Caucasian non-vegetarians and that protein intake correlates closely with the rate of antipyrine elimination. Nor are such dietary factors confined to the Western world: Fraser and his colleagues ${ }^{17}$ have also shown that cola nut consumption is one determinant of antipyrine half life in Gambian villagers; and frank malnutrition among adult Indians has also been reported ${ }^{18}$ to be associated with alterations in antipyrine elimination.

While the mechanisms underlying these changes are uncertain some of the practical implications are clearer. Both patients on reducing, high protein, or low carbohydrate diets and those who are debilitated, malnourished, or receiving prolonged intravenous infusions may have an altered capacity to metabolise drugs. Drug dosages should be tailored individually to meet the needs of these patients if treatment is to be safe and effective.

${ }^{1}$ Heading, R C, et al, British fournal of Pharmacology, 1973, 47, 415. 2 Prescott, L F, British fournal of Clinical Pharmacology, 1974, 1, 189. 3 Wagner, J G, et al, Nature, London, 1965, 207, 1301.

${ }^{4}$ Bianchine, J R, et al, Annals of the New York Academy of Sciences, 1971, $179,126$.

5 Lehmann, J, Acta Medica Scandinavica, 1973, 194, 181.

6 Gibaldi, M, Annals of the New York Academy of Sciences, 1971, 179, 19.

7 Wattenberg, L W, Cancer, 1971, 28, 99.

${ }^{8}$ Pantuck, E J, et al, fournal of Pharmacology and Experimental Therapeutics, 1976, 198, 278.

9 Conney, A H, et al, Clinical Pharmacology and Therapeutics, 1976, 20, 633.

${ }^{10}$ Smith, S E, and Rawlins, M D, Variability in Human Drug Response. London, Butterworth, 1973.

11 Vesell, E S, and Page, J G, Science, 1968, 161, 72.

12 Alexanderson, B, et al, British Medical fournal, 1969, 4, 764.

13 Whittaker, J A, and Price Evans, D A, British Medical fournal, 1970, 4, 323.

14 Vestal, R E, et al, Clinical Pharmacology and Therapeutics, 1975, 18, 425.

15 Kappas, A, et al, Clinical Pharmacology and Therapeutics, 1976, 20, 643.

${ }_{16}$ Fraser, H S, et al, Clinical Pharmacology and Therapeutics, 1977, in press.

17 Fraser, H S, et al, Clinical Pharmacology and Therapeutics, 1976, 20, 369.

18 Krishnaswamy, K, and Naidu, A N, British Medical fournal, 1977, 1, 538.

\section{Postoperative biliary stricture : falling frequency and improving results}

Over $90 \%$ of benign biliary strictures result from surgery in the upper abdomen, usually cholecystectomy. ${ }^{2}$ In the largest reported series, collected between 1940 and 1967 at the Lahey Clinic, ${ }^{3}$ there was no detectable mishap at the initial cholecystectomy in two-thirds of the 987 patients. The exact incidence of postoperative biliary stricture is difficult to determine, as most centres accumulate experience as a result of referral from elsewhere. Maingot ${ }^{4}$ estimates that it occurs in one out of 400-500 cholecystectomies. The skill and experience of the surgeon affect the incidence of biliary stricture, but other precipitating factors include the presence of fibrosis, adhesions, or acute inflammation in the area of operation. If removal of the gall bladder appears to be too difficult cholecystostomy should be carried out as an interim procedure. Recent figures are encouraging and suggest a roughly eightfold fall in the incidence of the problem between the 1950 s and the $1960 s^{5}$

Discharge from the wound, postoperative sepsis, prolonged biliary drainage, or jaundice are all early indications of stricture formation. Cholestasis or cholangitis, however, may be delayed for several years after the initial biliary surgery and may be associated with abdominal pain if calculi have developed proximal to a stricture as a result of stasis and infection. Much later, and only very rarely, variceal bleeding may occur when portal hypertension results from the development of secondary biliary cirrhosis. Cholangiography by the percutaneous transhepatic or endoscopic retrograde technique, or both, will show the full extent of a stricture and of the remaining normal extrahepatic duct and thereby help the surgeon in planning the best type of operative repair.

High strictures, affecting the common hepatic duct or right and left hepatic ducts, cause the greatest technical difficulties in repair. ${ }^{3}$ The bile is usually infected and this may also cause problems during or after operation. Success depends on a large stoma, apposition of mucosa throughout an anastomosis, and an adequate blood supply to, and no tension on, the suture line.$^{6} 7$ Plastic procedures to a slightly strictured duct are rarely possible, ${ }^{5}$ as are end-to-end anastomoses which should be performed only if less than $5 \mathrm{~mm}$ of duct substance has been lost. ${ }^{8}$ Most repairs require anastomosis of hepatic ducts to a jejunal loop ${ }^{3}$ or a Roux-en-Y procedure. ${ }^{2}$ 5-710

Indwelling tubes of varying design are generally used after operation, and some surgeons have advocated that splinting tubes should be left in situ for six to 12 months, during which fibrous tissue may mature without causing narrowing. ${ }^{3} 9$ Others, however, believe that prolonged intubation predisposes to both scarring and debris formation. ${ }^{25}$ Satisfactory results have been achieved for high anastomoses using a "mucosal graft" of a Roux loop splinted to each hepatic duct by a Y tube. ${ }^{11}$ The use of indwelling splints that pass through a hepaticojejunostomy, along the hepatic ducts, and thence through liver substance and out through the skin may also be helpful in selected cases. ${ }^{61012}$ Techniques now exist for changing these splinting tubes percutaneously, by guidewire or pull-through techniques, if they become obstructed with biliary concretions. ${ }^{512}$ In some patients, preliminary percutaneous tube drainage of dilated intrahepatic ducts may allow time for recovery of liver function and reduction in liver size before a planned repair procedure. ${ }^{2}$ 